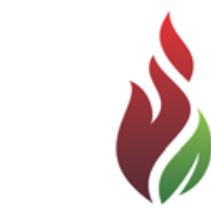

SUSTENERE

Publishing Corporation

\section{EFECTO DEL AGUA TRATADA MAGNÉTICAMENTE EN LA RECUPERACIÓN DE PLÁNTULAS DE ADENANTHERA PAVONINA EN ESTRÉS CLIMÁTICO}

\section{RESUMEN}

Este trabajo tuvo como objetivo evaluar la influencia del agua tratada bajo una inducción magnética constante de 0,12 T en la recuperación de las plántulas de Adenanthera pavonina, después de ser damnificadas por un huracán. El cultivo se realizó en invernadero, con sustrato compuesto por materia orgánica y arena (1:1); la desinfección y escarificación química de las semillas se efectuó con ácido sulfúrico (98 \%) y ácido bórico (5\%). Se evaluaron la longitud, el diámetro del tallo y el número de hojas por un período de 12 semanas. La aplicación del agua de riego tratada magnéticamente produjo un aumento estadísticamente significativo, en la longitud y el diámetro del tallo, no siendo así para la emisión de las hojas en esta fase de desarrollo. Los resultados confirmaron que el agua de riego, con tratamiento magnético de $0,12 \mathrm{~T}$, puede ser empleada como estimulante del crecimiento en esta especie forestal y en la recuperación de plantas dañadas por cambios climáticos.

PALABRAS-CLAVE: Adenanthera Pavonina, Magnéticamente Trata el Água, Huracán, Planta de Regeneración, Climate Exchange.

\section{EFEITO DA ÁGUA EM RECUPERAÇÃO MUDAS TRATADAS MAGNETICAMENTE ADENANTHERA PAVONINA STRESS NO CLIMA}

\section{RESUMO}

Este trabalho teve como objetivo avaliar a influência da água tratada sob indução magnética constante de 0,12 t na recuperação de mudas de Adenanthera pavonina depois de ser danificado por um furacão. A cultura foi realizada em estufa com substrato composto de matéria orgânica e de areia (1: 1); e desinfecção química escarificação semente foi feito com ácido sulfúrico $(98 \%)$ e ácido borónico $(5 \%)$ de ácido. Comprimento, diâmetro do caule eo número de folhas por 12 semanas foram avaliados. Aplicação de irrigação água tratada magneticamente produziu um aumento estatisticamente significativo no comprimento e diâmetro do caule, não para a emissão das folhas, nesta fase do desenvolvimento. Os resultados confirmaram que a água de irrigação com tratamento magnético de $0,12 \mathrm{~T}$, pode ser usado para impulsionar o crescimento nesta espécie de floresta ea recuperação de plantas danificadas pela mudança climática.

PALAVRAS-CHAVE: Adenanthera pavonina, Água Tratada Magneticamente, Furacão, Regeneração de Plantas, Câmbio climático.
Revista Ibero-Americana de

Ciências Ambientais, Aquidabã, v.5, n.2, Jun, Jul, Ago, Set, Out, Nov 2014.

ISSN 2179-6858

\section{SECTION: Articles}

TOPIC: Ecologia e Biodiversidade

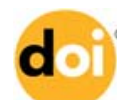

DOI: 10.6008/SPC2179-6858.2014.002.0001

Nádia Verçosa de Medeiros Rapôso Universidade do Estado do Amazonas, Brasil http://lattes.cnpq.br/5791520737203814 nadia.mestrinho@gmail.com

Yilan Fung Boix

Instituto de Ecología y Sistemática, Cuba http://lattes.cnpq.br/1238762710319411 yilan@cnea.uo.edu.cu

Clara Esther Martínez Manrrique Universidad del Oriente Cuba, Cuba clarita@cnea.uo.edu.cu

Albys Esther Ferrer Dubois Universidad del Oriente Cuba, Cuba http://lattes.cnpq.br/2928079258400345 albys@cnea.uo.edu.cu

Guillermo Asanza Kindelan Universidad del Oriente Cuba, Cuba asanza@cnea.uo.edu.cu

Fidel Gilart González Universidad del Oriente Cuba, Cuba fgilart@cnea.uo.edu.cu

Received: 18/05/2014

Approved: 15/11/2014

Reviewed anonymously in the process of blind peer.

\section{Referencing this:}

RAPÔSO N.V.M : BOIX Y F : MANRRIQUE, C.E.M. DUBOIS, A. E. F. D.; KINDELAN, G. A.; GONZÁLEZ, F. G. Efecto del agua tratada magnéticamente en la recuperación de plántulas de adenanthera pavonina en estrés climático. Revista Ibero-Americana de Ciências Ambientais, Aquidabã, v.5, n.2, p.6-17, 2014. DOI: http://dx.doi.org/10.6008/SPC21796858.2014.002.0001 


\section{INTRODUCCIÓN}

Actualmente se han dedicado espacios a los efectos de los cambios climáticos debido a la ocurrencia de un elevado número de eventos meteorológicos cada vez más violentos, entre los que se encuentran los huracanes, sequías e inundaciones. El mantenimiento de los bosques permite estabilizar el clima, la absorción del dióxido de carbono $\left(\mathrm{CO}_{2}\right)$ y preserva el equilibrio atmosférico.

La degradación ambiental debido a la deforestación desordenada en los países de la región amazónica, es uno de los factores responsable de los cambios climáticos, que amenaza las especies de la fauna y flora y las pone en peligro de extinción (efecto dominó). La interacción entre los sistemas biológicos, físicos, sociales y económicos demuestra que los bosques amazónicos están perdiendo espacios producto de los impactos debido a los cambios climáticos (BUSTAMANTE et al., 2006; JAIME et al., 2011). La capacidad natural de estos bosques de superar una situación crítica y regresar a su estado natural original, está siendo limitada por la frecuencia con que ocurren estas agresiones ambientales. Los mecanismos que permiten a un ecosistema retornar a su punto de equilibrio tienen un límite, que puede ser sobrepasado, dependiendo del grado de perturbación que el hombre provoque en él (BUSTAMANTE et al., 2006; JAIME et al., 2011). Esta situación exige que se intensifiquen las investigaciones para conocer los diferentes procesos físicos, químicos y biológicos relacionados con el funcionamiento extremadamente complejo de estos bosques. Otro problema presente en la mayoría de las especies forestales amazónicas, son sus semillas con tegumentos duros e impermeables, que restringe la entrada de agua y oxígeno, ofreciendo una alta resistencia física al crecimiento del embrión, retardando la germinación por largos períodos, dificultando la recuperación de bosques (SANTOS et al., 2004). Por otro lado, el desarrollo de las plántulas a partir de las técnicas tradicionales de reproducción vegetativa es afectado por su difícil enraizamiento.

La especie Adenantera pavonina L. al ser introducida en Brasil se difundió en todos los estados, principalmente en la región norte, donde se encuentra bien adaptada. Esta especie se adapta a cualquier tipo de suelo y no es exigente en cuanto a las condiciones climáticas. Su cultivo se extiende desde suelos ricos en nutrientes a pobres y arenosos. Posee múltiples usos, pudiendo ser utilizada en proyectos de reforestación, paisajísticos como planta ornamental o para dar sombras, en la artesanía o como forrajera (AKKASAENG, 1989). Las semillas y madera han sido utilizadas en control biológico como bioinsecticida, ya que inhiben las enzimas digestivas de lepidópteros. Presenta un tamaño que varía de 15 a 20 metros de altura, copa redondeada, floración amarilla clara y su crecimiento es rápido, por esto es considerada como planta pionera (FONSECA \& ANDRADE, 2001).

Cualquier estudio que contribuya a viabilizar la reproducción vegetativa y aumentar la velocidad en la reposición de las especies forestales es de importancia y de gran interés en el manejo adecuado de los bosques. Una forma de lograr este propósito, es el uso del riego con agua tratada magnéticamente en la producción de plántulas. 
A pesar de ser pocos los estudios de la aplicación de los campos magnéticos y el uso del agua tratada magnéticamente en especies forestales, actualmente se intensifican las investigaciones de los efectos del agua tratada magnéticamente para mejorar la producción agrícola. Se tiene conocimiento que los campos magnéticos estáticos con bajas inducciones magnéticas influyen en los procesos bioquímicos y fisiológicos, mejorando la germinación de las semillas, así como los rendimientos en las cosechas, sin afectar al medio ambiente. El agua tratada magnéticamente induce alteraciones favorables, estimula algunos procesos morfológicos, fisiológicos y bioquímicos, facilitando la absorción de nutrientes (KRAMER, 1982; ROSEN, 1992; VALBERG, 1995; RIOJA, 1999; TENDERFORD, 2003). Varias hipótesis han sido propuestas para explicar los efectos biológicos que ejerce el campo magnético en los organismos vivos, entre ellos se incluyen la modificación de la permeabilidad de la membrana, el flujo de iones, la orientación de partículas ferromagnéticas (magnetosomas), los efectos sobre los procesos enzimáticos (metabolismo) y sobre la división celular (IBÁÑEZ, 1989; POLCK \& POSTOW, 1996; BARNES \& GREENEBAUM, 2006).

Diferentes estudios concluyen que el tratamiento magnético del agua tiende a prolongar el ciclo de desarrollo de varias especies de plantas retardando el envejecimiento, anticipando la floración y la fructificación. Un posible mecanismo de la acción del campo magnético puede ser debido a la modificación en la estructura molecular del agua, que facilita su absorción por las plantas (VÉLEZ \& PIROVOROVA, 1993). Se considera que ésta agua es mejor asimilada por las células, causando modificaciones en los diferentes potenciales, que se establecen durante el proceso de ósmosis, favoreciendo la intensidad del flujo de agua en el suelo, siendo más rápido que en condiciones normales. A pesar de estos beneficios, no existen estudios ni teorías concluyentes que demuestren los efectos de los campos magnéticos estáticos y electromagnéticos en los procesos fisiológicos, bioquímicos y moleculares del metabolismo de las plantas.

En la mayoría de las especies forestales y principalmente las amazónicas, los estudios relacionados con la propagación, fisiología y crecimiento de plantas forestales son escasos. El agua tratada magnéticamente en el riego puede ser utilizada por los agricultores como una herramienta en la producción y conservación de plántulas, con el fin de incrementar y recuperar el número de especies.

Debido a la gran pérdida de árboles por causa del huracán Sandy en Santiago de Cuba y conociendo la importancia de los efectos de los campos magnéticos en el crecimiento de especies vegetales, con base en la hipótesis que el riego con agua tratada magnéticamente provoca un incremento en el crecimiento de la longitud y diámetro del tallo, así como de la emergencia de hojas, el presente trabajo tiene como objetivo evaluar el comportamiento del crecimiento y desarrollo de las plantas que fueron afectadas por un huracán, a través de las variables morfológicas longitud y diámetro del tallo, así como emergencia de las hojas.

\section{MÉTODOS}


Cuadro 1: Características del sustrato.

\begin{tabular}{|c|c|c|}
\hline Macroelementos & $\begin{array}{c}\text { Microelemento } \\
\text { mg kg-1 }^{-1}\end{array}$ & Propiedades físicas y químicas \\
\hline $\begin{array}{l}\text { Materia orgánica }>4,3 \% \\
\mathrm{~K}^{+}(0,60 \%) \\
\mathrm{Na}^{+}(0,18 \%)\end{array}$ & $\begin{array}{l}\mathrm{Fe}(38.966,20) \\
\mathrm{Al}(34.723,80) \\
\mathrm{V}(4,44)\end{array}$ & $\begin{array}{l}\mathrm{P}_{2} \mathrm{O}_{5}\left(46 \mathrm{mg} \mathrm{L}^{-1}\right) \\
\mathrm{K}_{2} \mathrm{O}\left(53,2 \mathrm{mg} \mathrm{L}^{-1}\right) \\
\mathrm{MgO}\left(11,09 \mathrm{mg} \mathrm{L}^{-1}\right)\end{array}$ \\
\hline $\mathrm{Ca}^{++}\left(31.178 \mathrm{mg} \mathrm{kg}^{-1}\right)$ & $\mathrm{Cr} \mathrm{Cr}(13,66)$ & Conductividad eléctrica: $273 \mu S$ \\
\hline $\mathrm{Mg}^{++}\left(10.677 \mathrm{mg} \mathrm{kg}^{-1}\right)$ & $\begin{array}{l}\text { Co }(18,34) \\
\mathrm{Cd}(6,86) \\
\mathrm{Pb}(85,01) \\
\mathrm{Ni}(12,46) \\
\mathrm{Cu}(114,62) \\
\mathrm{Zn}(108,49)\end{array}$ & $\mathrm{pH}: 6,4$ \\
\hline
\end{tabular}

Para el tratamiento magnético del agua de riego, se utilizó un acondicionador magnético de imanes permanentes de $20 \mathrm{~cm}$ de longitud, con inducción de 0,12 T diseñado, construido y caracterizado en el CNEA, con el uso de un gausímetro de Lake Shore, modelo 410, de referencia internacional.

El agua tratada magnéticamente (ATM) presentaba las características química que se muestran en el cuadro 2. Se comenzó a utilizar, durante 30 minutos, a partir del séptimo día después de la catástrofe natural debido a la destrucción de las instalaciones (octubre 2012).

Cuadro 2: Características químicas del agua de riego.

\begin{tabular}{|c|c|c|c|}
\hline Composición mineral & Iones & $\mathrm{mg} \mathrm{L}^{-1}$ & Propiedades físicas \\
\hline \multirow{5}{*}{$\begin{array}{l}\mathrm{HCO}_{3}(261,2) \\
\mathrm{Cl}^{-}(33,18)\end{array}$} & $\mathrm{Na}^{+}$ & 12,42 & Conductividad eléctrica: $0,25 \mathrm{~S} \mathrm{~cm}^{-1}$. \\
\hline & $\mathrm{Ca}^{+2}$ & 25,60 & $\mathrm{pH} \mathrm{7,87}$ \\
\hline & $\mathrm{Mg}^{+2}$ & 6,72 & Dureza cálcica (144,6 mg L-1) \\
\hline & $\begin{array}{l}\mathrm{KI} \\
\mathrm{HCO}_{3}{ }^{-} \\
\mathrm{Cl}^{-} \\
\mathrm{SO}_{4}^{-2} \\
\mathrm{CO}_{3}{ }^{-2}\end{array}$ & $\begin{array}{r}4,68 \\
73,20 \\
17,75 \\
9,61 \\
18,00 \\
\end{array}$ & $\begin{array}{l}\text { Dureza magnésica }\left(79,95 \mathrm{mg} \mathrm{L}^{-1}\right) \\
\text { Dureza total }\left(224,5 \mathrm{mg} \mathrm{L}^{-1}\right)\end{array}$ \\
\hline & Total & 118,56 & \\
\hline
\end{tabular}

Caracterización de las variables. Se evaluaron las variables morfoanatómicas longitud y diámetro del tallo, además del número de hojas. Las mediciones biométricas fueron efectuadas una vez por semana durante 12 semanas, se empleó una regla graduada $(\mathrm{cm})$, para medir la longitud y un pie de rey $(\mathrm{mm})$ para el diámetro. El tamaño de muestra fue de 20 plantas para cada tratamiento, con tres réplicas.

Análisis estadístico. Para el análisis estadístico, se empleó la prueba t, para evaluar hipótesis específicas acerca de la diferencia entre las medias de las poblaciones de las cuales provienen las dos muestras. Todos los análisis se hicieron con el paquete estadístico Statgraphics Plus 5.1.

\section{RESULTADOS}

En la longitud del tallo, se observó que las plántulas regadas con agua tratada magnéticamente con inducción magnética de $0,12 \mathrm{~T}$, tuvieron diferencias estadísticamente 
significativas $(P<0,05)$ en relación a las plántulas control. Alcanzando valores medios para la longitud del tallo de $22,16 \mathrm{~cm} \pm 0,68$ y de $19,46 \mathrm{~cm} \pm 1,10$ para el control (figura $1 \mathrm{~A}$ ).
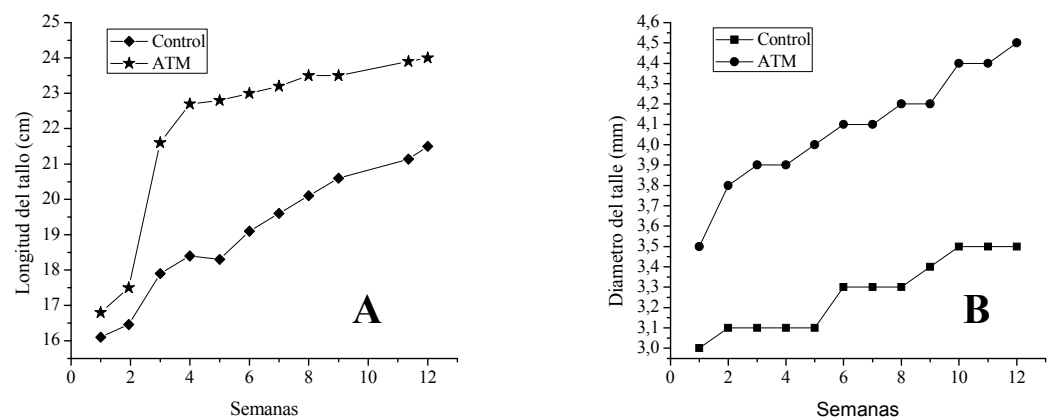

Figura 1: Comportamiento de la longitud $(A)$ y del diámetro del tallo $(B)$ en $A$. pavonina regada con agua tratada magnéticamente.

Para el diámetro de tallo (figura $1 \mathrm{~B}$ ), se pudo comprobar estadísticamente $(P<0,05)$ que la media del crecimiento en diámetro de las plantas fue mayor en las tratadas $(4,1 \mathrm{~mm} \pm 0,13)$, que en las no tratadas $(3,3 \mathrm{~mm} \pm 0,17)$. El periodo de estabilización en aquellas sin tratamiento varió de 2 a 5 semanas, mientras que las regadas con agua tratada magnéticamente fue de una semana. tratamiento magnético aplicado aceleró el crecimiento, como se pudo comprobar estadísticamente $(P<0,05)$.

Para la variable número de hojas (figura 2), el análisis estadístico no mostró diferencias entre las medias de los dos tratamientos $(P>0,05)$. Sin embargo, desde el punto de vista biológico los mayores valores se encontraron en las plantas que fueron regadas con agua tratada magnéticamente $(5,14 u \pm 0,27)$, en relación al control $(4,88 u \pm 0,26)$, lo cual permitió que las plantas tuvieran una mayor supervivencia y con ello una mayor adaptación y mejor actividad fisiológica luego que pasaron por un estrés ambiental como el huracán.

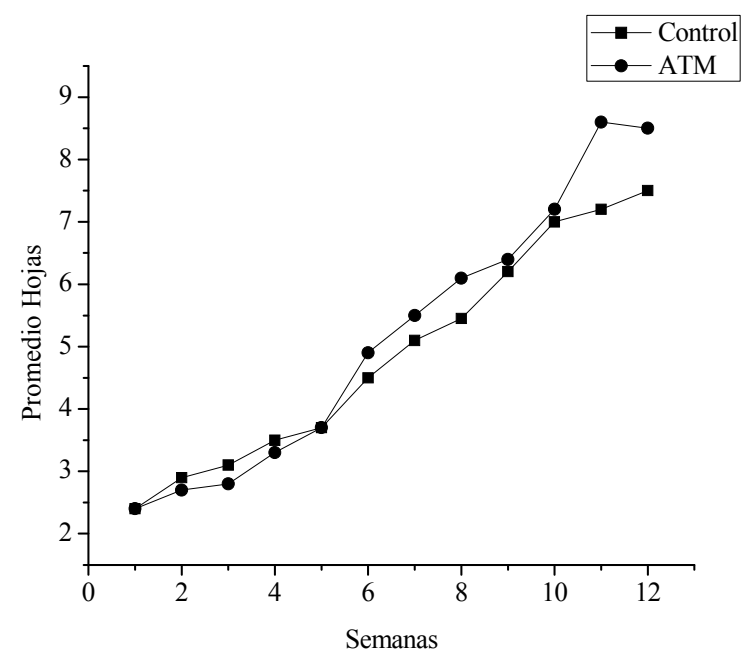

Figura 2: Comportamiento del emisión de hojas en posturas de $A$. pavonina regadas con agua tratada magnéticamente.

\section{DISCUSIÓN}


Las diferencias estadísticamente significativas en el desarrollo de la altura y diámetro del tallo de las plantas regadas con agua tratada magnéticamente (inducción 0,12 T), con relación al control (figuras $1 \mathrm{~A}$ y $1 \mathrm{~B}$ ), ocurren porque fisiológicamente el crecimiento de las plantas no tiene lugar de manera uniforme, está concentrado en los meristemos, debido al incremento enzimático y al aumento de la eficiencia de los procesos relacionados con la división celular.

El efecto de magnetización es considerado como un efecto biológico primario, responsable de la alteración en la permeabilidad de la membrana celular, permitiendo una mayor frecuencia de cambios en la polarización de la misma, favoreciendo la apertura de los poros y canales, lo que posibilita mayor absorción de nutrientes, que estimulan el desarrollo del tallo (CARBONELL \& MARTÍNEZ, 1996).

Como se conoce, el genotipo es el factor más importante para la supervivencia de cualquier especie, lo cual influye en una mejor adaptación a condiciones adversas (DEBERGH, 1991). Varias investigaciones coinciden con la acción favorable del agua tratada magnéticamente en el desarrollo de la longitud del tallo de varias especies vegetales. Trabajos realizados por Justiz et al. (1995), demostraron incrementos en la longitud total de plántulas de Zeas mays colocadas a germinar, utilizando agua tratada magnéticamente con inducción de 0,15 T.

Los efectos beneficiosos del tratamiento magnético al agua pueden deberse a algunas modificaciones en las plantas a nivel bioquímico y sus posibles efectos en el nivel celular. Se ha demostrado la influencia de los campos eléctricos y magnéticos externos en la activación de iones y la polarización de los dipolos en células vivas (MOON \& CHUNG, 2000). Los campos electromagnéticos (EMF) pueden alterar la estructura de la membrana plasmática así como su función (PARADISI et al., 1993). Basant y Harsham (2009) emplearon un dispositivo para el tratamiento al agua de riego en un intervalo de campo magnético de 3,5 a $136 \mathrm{mT}$. El análisis de los datos sugirieron que los efectos de tratamiento magnético varían con el tipo de planta y de agua de riego utilizada, también obtuvieron aumentos estadísticamente significativos en el rendimiento y la productividad de la planta.

Los cambios en las características físico-químicas del agua sometida a tratamiento magnético, afectan principalmente a la disolución y absorción de nutrientes por las raíces. El aumento de la utilización de reservas y mayor absorción de agua por las raíces en Raphanus sativus L. y Hordeum sativum fue demostrada por Smith (1993).

Podleoeny et al. (2004) estudiaron los efectos del tratamiento magnético con la exposición de las semillas de Vicia faba L. antes de la siembra y observaron importantes efectos beneficiosos sobre la germinación de las semillas, la tasa de aparición, rendimiento y emergencia de las mismas a los 2-3 días en comparación con el tratamiento control.

Las investigaciones relacionadas con el agua tratada magnéticamente en el riego para la agricultura, demuestran que las plantas utilizan menor dosis de riego, crecen más vigorosas que las plantas sin tratar (AMAYA, 1995; CARBONELL \& MARTíNEZ, 1996). Este hecho, es comprobado 
por Castillo Mirales (2008) y Ferrer (2005), en Lycopersicum esculentum M. y Cucumis sativus L. var SS-5 respectivamente, donde lograron un mayor crecimiento de la longitud del tallo para las plantas que fueron regadas con agua tratada magnéticamente. Fung et al. (2008) emplearon tratamientos con un rango de inducción de 0,06 T y 0,12 T, además de un control; obteniendo que las plantas regadas con agua tratada magnéticamente tuvieron un mejor crecimiento y desarrollo en la especie Rosmarinus officinalis L. Resultados análogos de desarrollo en la altura y diámetro del tallo fue obtenido por Pérez (1993), en el cultivo de Cucumis sativum. Especies de Lens culinaris L. y Helianthus annuus L. fueron sometidas a la acción de campos magnéticos las cuales mostraron una mayor longitud y diámetros de los tallos (MARTíNEZ, 1997).

Los resultados obtenidos en A. pavonina corroboran los encontrados por Novoa (2004), quien aclimató plántulas de Musa sp. var. FHIA-18 con agua tratada magnéticamente, logrando un $98 \%$ de supervivencia con respecto a un $89 \%$ para las plantas control. Estas evidencias respaldan los efectos del riego con agua tratada magnéticamente en la supervivencia de especies vegetales.

Para crecer y sobrevivir, las plantas utilizan el agua como fuente principal para incorporar minerales a través del suelo; el agua, al ser excitada o perturbada bajo la acción de un campo magnético, provoca que su estructura tenga modificaciones, lo que permite que esta sea mejor asimilada o absorbida por las células. En el presente experimento, el riego con agua tratada magnéticamente tuvo un efecto positivo en la recuperación de las plantas de A. pavonina. Todo parece indicar que el agua, cuando recibe tratamiento magnético, es perturbada en su estructura (CHANG, 2006), ya que la aplicación de campos magnéticos externos en el agua, en intensidades de 1 a $10 \mathrm{~T}$, inducen alteraciones en los aglomerados, variando la estructura y tamaño de estos. Zhou et al. (2000) reportan que el desorden de las moléculas es mayor por debajo de $1 \mathrm{~T}$, lo cual puede estar relacionado con la obtención de un agua más simple.

La apertura y el cierre de las acuaporinas para el paso del agua a través de la membrana regulan el volumen celular en las plantas y las mismas participan en la elongación celular, también pueden estar moduladas por señales moleculares, que reciben y condicionan estímulos eléctricos del orden de los milisegundos, como ocurre con otros transportadores. Es evidente que estas señales pueden asociarse a la luz, lo cual posibilita el incremento del contenido de agua en base a la presencia de protones que generan en potencial reductor NADPH (VERA-ESTRELLA et al., 2005).

Estos efectos pueden explicar los resultados obtenidos en este trabajo, con el uso del agua de riego tratada magnéticamente, donde se obtuvo un aumento del tamaño y diámetro del tallo. En este caso, el movimiento de agua a través de la bicapa lipídica por los poros que forman las acuaporinas pudo incrementarse. Un aumento del contenido hídrico va acompañado de la turgencia y alongamiento celular, lo que permite la apertura de los estomas y el desarrollo de la transpiración como eventos fisiológicos de gran importancia para el metabolismo de las plantas. 
Los análisis de los resultados obtenidos en este trabajo, al ser comparado con los obtenidos en otras investigaciones, también pueden afirmar la influencia que ejerce el agua tratada magnéticamente sobre los procesos fisiológicos de desarrollo que realizan las plántulas $A$. pavonina. Galland y Pazur (2005) hacen una revisión bibliográfica y observan que, en el pasado, las investigaciones fisiológicas eran efectuadas de manera poco sistemática y sin explicación de los efectos del campo magnético, pues los resultados de la mayoría de los estudios permanecían en un nivel fenomenológico, caracterizado por el desconocimiento de los mecanismos, a pesar de que la física argumentó varias teorías que servían como paradigmas para la magnetorecepción.

En la última década, se han intensificado las investigaciones de las especies vegetales en relación a los campos geomagnéticos, magnéticos estáticos e electromagnéticos como por ejemplo, en Allium cepa, Novitsky et al. (2001) utilizando inducción de 505 T observaron en las hojas un crecimiento en la concentración de clorofila y proteínas. Isaac et al. (2004), demuestran los efectos positivos de los campos magnéticos en el desarrollo del estaquillado de Psidium guajava L.

En semillas de Lactuca sativa, al aplicar campos magnéticos con inducción de 0-10 de mT, aumentó el contenido de agua (GARCÍA-REINA et al., 2001). En Lens culinaris, Glycine soja y Triticum aestivum, Peñuelas et al. (2004) aplican campos magnéticos con inducción de 17,6 mT e inhibieron el crecimiento de la raíz en las tres especies. En Zea mays, al aplicar campos magnéticos con inducción de $150 \mathrm{mT}$ en las semillas se estimularon la germinación y el crecimiento de las plántulas (ALADJADJIAN, 2002). Lo mismo ocurrió cuando Carbonell et al. (2000) aplicaron una inducción de $150 \mathrm{mT}$ y $250 \mathrm{mT}$ para estimular la germinación y el crecimiento de la plántula de Oryza sativa y Linum bienne con aplicación de 0,1 nT - $1 \mu \mathrm{T}$, Belova y Lednev (2000ab) modularon el geotropismo en Helianthus annuus y Triticum aestivum. Fischer et al. (2004) utilizaron dos inducciones magnéticas de $20 \mu \mathrm{T}$ y $16 \mathrm{~Hz}$, logrando un aumento del peso fresco y de la germinación. Bauréus Koch et al. (2003) realizaron la modulación del $\mathrm{Ca}^{+2}$ importado en membranas separadas.

Estos resultados demostrados por numerosos científicos, corroboran la utilidad en el uso del agua de riego tratada magnéticamente para el desarrollo de la especie $A$. pavonina, lo que a su vez depende de la inducción magnética y tiempo de exposición del campo magnético utilizado; todo lo cual posee un efecto adaptativo que permite la recuperación en mayor medida de las plantas afectadas por el severo fenómeno natural producido por los cambios climáticos. Así es posible dar una explicación de los mecanismos de acción del riego con agua tratada magnéticamente en el cultivo de plantas leñosas dañificadas por estrés climático como sucede para las plántulas de $A$. pavonna, las que se recuperan más rápido que los controles, al utilizar agua tratada magnéticamente lográndose mayor aumento en la longitud y diámetro del tallo.

\section{CONCLUSIONES}


De manera general, los resultados sugieren que el uso del agua tratada magnéticamente tiene un efecto positivo en la altura y el diámetro del tallo, así como en el número de hojas de $A$. pavonina. Ello favorece que las plantas se recuperen después de ser afectadas por un huracán. Por ello, el uso del riego con agua tratada magnéticamente puede ser una alternativa para la recuperación de plantas en áreas afectadas por huracanes.

\section{AGRADECIMIENTOS}

A Dios, que me permitió realizar este trabajo sosteniéndome y estando conmigo en los días difíciles. Al Gobierno del Estado do Amazonas, que posibilitó la realización de este estudio. Al Profesor MSc. Mário Augusto Figueiredo Bessa director de la Escuela Superior de Tecnología de la Universidad de Estado de Amazonas (EST/UEA). Al Centro Nacional de Electromagnetismo Aplicado (CNEA) de la Universidad de Oriente en Santiago de Cuba, en especial al equipo de Laboratorio de Biotecnología Vegetal.

\section{REFERENCIAS}

AKKASAENG, R.. Evaluation of trees and shrubs for forage and fuelwood in Northeast Thailand. International Tree Crops Journal, n.5, p.209-220, 1989

ALADJADJIYAN, A.. Study of the influence of magnetic field on some biological characteristics of Zea mays. Journal of Central European Agriculture, n.3, v.2, p.89-94, 2002.

AMAYA, J.. Incidencia de campos magnéticos estacionarios en la germinación y crecimiento de semillas. Agricultura, n.761, p.1049-1054, 1995.

BARNES F.; GREENEBAUM, B.. Bioengineering and biophysical aspects of electromagnetic fields. Handbook of Biological Effects of Electromagnetic Fields. 3 ed. CRC Press, Boca Raton, 2006.

BASANT, L. M.; HARSHARN, S. G.. Magnetic treatment of irrigation water: Its effects on vegetable crop yield and water productivity. Agricultural Water Management, n.96, p.1229-1236, 2009.

BAURÉUS KOCH, C. L. M,; SOMMARIN, M.; PERSSON, B. R. R.; SALFORD, L. G.; EBERHARDT, J. L.. Interaction between weak low frequency magnetic fields and cell membranes. Bioelectromagnetics, p.395402, 2003.

BELOVA, N. A.; LEDNEV, V. V.. Activation and inhibition of gravitropic reaction of plants using weak combined magnetic fields. Biofizika, p.1102-1107, 2000.

BUSTAMANTE, M. M. C.; CORBEELS, M.; SCOPEL, E.; ROSCOE, R.. Soil carbon sequestration potential in the cerrado region of Brazil. In: LAL, R.; CERRI, C. C.; BERNOUX M.; ETCHEVERS J.. Carbon sequestration in soils of Latin America. The Haworth Press, 2006.

FONSECA, S. C. L.; PERES, G. A.. Germinação de sementes de olho-de-dragão (Adenanthera pavonina L.): ação de poliaminas na atenuação do estresse salino. Revista Brasileira de Sementes, v.23, p.14-20, 2001.

CAMPANIONI, N.; PEÑA, E.; OJEDA, Y.; GARCÍA, M.; CARRIÓN, M.; GONZÁLEZ, R.; DÍAZ, M.; NAVARRO, A.. Tecnología de producción para plántulas en cepellón. INIFAT, Havana, 2002.

CARBONELL, M.; MARTÍNEZ E.. Estudio de la solubilidad de distintos compuestos en agua imantada. Química e Industria, 1996. 
CARBONELL, E.; MARTINEZ, J.; AMAYA, M.. Stimulation of germination of rice by a static magnetic field. Electro-Magnetobiol, n.19, v.1, p.121-128, 2000.

MIRALES, M. E. C.. Evaluación de la germinación, supervivencia y rendimiento de Lycopersicon esculentum M. con agua tratada magnéticamente. Trabajo de Titulación (Licenciado en Ciencias Biológicas) - Facultad des Ciencias Naturales, Santiago de Cuba, 2008.

CHANG, K. T.; WENG, C. I.. The effects and external magnetic field on the strutural of liquid water using molecular dimanycs simulations. Journal of Applied Physics, n.100, 2006.

DEBERGH, P.. Aclimatization techniques of plant from in vitro. Acta Horticulturae, n.289, p.291-300, 1991.

FERRER, A.. Utilización de riego con tratamiento magnético en el crecimiento de pepino (Cucumis sativus L.). Revista Tecnología Química, 2005.

FISCHER, G.; TAUSZ, M.; KOCK, M.; GRILL, D.. Effects of weak $162 / 3 \mathrm{~Hz}$ magnetic fields on growth parameters of young sunflower and wheat seedlings. Bioelectromagnetics, n.25, v.8, p.638-641, 2004.

FUNG. Y.: ISAAC, E.; FERRER, A,; BOTTA, A.M.. Riego con agua tratada magnéticamente en Rosmarinus officinalis L. (romero) como alternativa en la propagación convencional. Centro Agrícola, n.35 v.1, p.23-27, 2008.

GALLAND, P.; PAZUR, A.. Magnetoreception in plants. J Plant Res, n.118, p.371-389, 2005.

GARCÍA-REINA, F.; PASCUAL, L.; FUNDORA, I. A.. Influence of a stationary magnetic field on water relations in lettuce seeds. Bioelectromagnetics, n.22, v.8, p.596-602, 2001.

IBÁÑEZ, J.. Bases biológicas de los efectos de los campos magnéticos, en tribuna médica. Hospitales Montoya, Madrid, n.40, p.34-36,1989.

ISAAC, E.; FERRER, A.; FUNG, Y.; NOVOA, I.. Influencia de los campos magnéticos en el estaquillado de guayaba enana (Psidium guajava $L$.). Simposio Internacional de Ciencias Biológicas Charles T. Ramsden, 2004.

JAIME, J. V.. S.O.S. AMAZONÍA. Editorial de Ciencias Sociales, Havana, p.1-3, 2011.

JUSTIZ, E.; FERRER, A.; NOVOA, I.. Influencia del tratamiento magnético del agua en la germinación de semillas de maíz (Zea mays). Forum Nacional de Electromagnetismo Aplicado. Santiago de Cuba (Centro Nacional de Electromagnetismo Aplicado.Universidad de Oriente), 1995.

KRAMER, P.. Water and plant productivity of yield. Handbook of Agricultural Productivity, Florida, 1982.

MARTÍNEZ, E.. Respuesta biológica del desarrollo de plantas de cultivo a la acción de campos magnéticos estacionarios. Alcalá de Henares, Madrid, p.41-50., 1997.

MOON, J. D.; CHUNG, H. S.. Acceleration of germination of tomato seeds by applying AC electric and magnetic fields. Journal of Electrostatics, n.48, p.103-114, 2000.

NOVITSKY, Y. I.; NOVITSKAYA, G. V.; KOCHESHOVA, T. K.; NECHIPORENKO, G. A.; DOBROVOL'SKII, M. V.. Growth of green onions in a weak permanent magnetic field. Russ J Plant Physiol, n.48, p.709-715, 2001.

NOVOA, I.. Efectos del agua tratada magnéticamente en la aclimatización de plátano (FHIA-18). Instituto Nacional de Ciencia Agrícolas, 2004.

PARADISI, S.; DONELLI, G.; SANTINI, M. T.: STRAFACE, E.; MALORNI, W. . A 50-Hz magnetic field induces structural and biophysical changes in membranes. Bioelectromagnetics, n.14, p.247-255, 1993.

PEÑUELAS, J.; LLUSIÀ, J.; MARTíNEZ, B.; FONTCUBERTA, J.. Diamagnetic susceptibility and root growth responses to magnetic fields in Lens culinaris, Glycine soja, and Triticum aestivum. Electromagnetic Biology and Medicine, n.23, v.2, p.97-1 12, 2004. 
PÉREZ, R.. Efecto del tratamiento magnético del agua de riego sobre el desarrollo del cultivo de Pepino (Cucumis sativum) y la incidencia de plagas y enfermedades. Santiago de Cuba, 1993.

PODLEOENY, J.; PIETRUSZEWSKI, S.; PODLEOENA, A.. Efficiency of the magnetic treatment of broad bean seeds cultivated under experimental plot conditions. International. Agrophysics, n.18, v.65-71, 2004.

POLK, C.: POSTOW, E.. Handbook of biological effect of electromagnetic fields. 2 ed. CRC Press LLC, 1996.

RIOJA, J.. Electroterapia y Electrodiagnóstico. 2 ed. Valladolid, 1996.

ROSEN, A.D.. Membrane response to static magnetic fields: effect of exposure duration. Biochimical and Biophysical Acta, v.1148, p.317-20, 1992.

SANTOS, T.O.; MORAIS, T.G.O.; MATOS, V. P. . Escarificação mecânica em sementes de chicá (Sterculia foetida L.). Revista Árvore, n.28, p.1-6, 2004.

SMITH, S. D.; MCLEOD, B. R.; LIBOFF, A. R.. Effects of CR-tuned $60 \mathrm{~Hz}$ magnetic fields on sprouting and early growth os Raphanus sativus. Bioelectrochemical Bioenergy, n.32, p.67-76, 1993.

TENDERFORD, T.. The wonders of Magnetism. Bioelectromagnetic, n.24, v.1, p.3-11, 2003.

VALBERG, A.. Designing EMF experiments: what's required to characterize "exposure"? Bioelectromag, v.16, p.396-401, 1996.

VÉLEZ R.; PIROVOROVA, N.. Aplicación de campos magnéticos en líquidos y soluciones acuosas. Conferencias. Santiago de Cuba, 1993.

VERA-ESTRELLA, R.; BARKLA, B. J.; PANTOJA, O.. La biología y la fisiología de las acuaporinas de plantas. biojournal.net., n. 1, 2005.

ZHOU, K. X.; LU, G. W.; ZHOU, Q. C.; SONG, J. H.; JIANG, S. T.; XIA, H. R.. Monte Carlo simulation of liquid water in a magnetic field. Journal of Applied Physics, n.1802, 2000. 